\title{
Editorial
}

\section{Health problems of pesticide usage in the Third World}

Pesticides are important to the life of the people and governments of Third World countries where they are extensively used both in agriculture and in public health programmes. Approximately $60 \%$ of the workforce of these countries are employed in agriculture $^{1}$ and pesticides are used to control pests that are estimated to devastate at least a third of the potential crops in the countries in the Third World, ${ }^{2}$ so that benefits result from increased crop yields.

In the field of public health great suffering results from vector borne diseases. It is estimated that malaria results in some 150 million clinical cases a year whereas filariasis affects about 250 million people and schistosomiasis causes debilitating and sometimes fatal disease in some 250 million people. The eradication of these and other vector borne diseases depends to a great extent on the use of pesticides.

This then is the promise of pesticides but their use is, at the same time, associated with hazards to human health.

It is important to appreciate that the type and extent of health hazards arising from the use of pesticides and their consequent impact on national health priorities in the countries of the Third World differ from those encountered in the industrialised countries. Though the industrialised countries use $80 \%$ of the world's agrochemicals, ${ }^{3}$ they probably suffer only $1 \%$ or less of all deaths due to acute pesticide poisoning, so that acute pesticide poisoning is not a major health problem in the countries of the industrialised world. The same is not necessarily true for the Third World. Firstly, accurate data on the extent of acute pesticide poisoning in these countries are lacking, which is why WHO had to use a mathematical model to estimate the problem. ${ }^{4}$ On the basis of this model, WHO believe that about 500000 cases of pesticide poisoning resulting in at least 5000 deaths occur each year. These figures are probably an underestimate. Recent studies showed that in Sri Lanka (population around 14.5 million) there were about 13000 hospital admissions each year for acute pesticide poisoning, resulting in about 1000 deaths. $^{5}$ The extrapolation of these figures to the countries of the developing world would suggest that about 2.9 million cases of acute pesticide poisoning requiring admission to hospital with about 220000 deaths are likely to occur annually. Though the Sri Lankan study observed that only $25 \%$ of the cases of acute pesticide poisoning resulted from occupational and non-occupational accidents, this percentage nevertheless constitutes a large number of people. An indication of its importance as an occupational health problem in Sri Lanka is the estimate that each year five of every 1000 agricultural workers are admitted to hospital for acute pesticide poisoning. These figures indicate that acute pesticide poisoning is a high priority health issue in Sri Lanka as well as in other countries of the Third World.

To achieve effective control of acute pesticide poisoning in these countries the extent of the problem must be established to provide data for the national decision makers to use to set priorities and take appropriate corrective action. For instance, in Sri Lanka in the year when about 1000 deaths occurred from acute pesticide poisoning only 572 deaths were recorded as being due to poliomyelitis, diphtheria, tetanus, and whooping cough; not a single death occurred from malaria. But the anomaly is that whereas health expenditure to control malaria is substantial, virtually no budgetary allocation exists for the control of acute pesticide poisoning.

It is appropriate to consider some of the factors that contribute to occupational and other accidental pesticide poisoning. Studies in Sri Lanka have shown that most farmers are aware of the simple precautionary measures necessary to prevent the hazards arising from pesticide use but were unable to put this knowledge into practice. ${ }^{6}$ For example, although aware that they should not use leaking knapsack sprayers, the farmers continued to use them and this was one of the commonest causes of poisoning. Often the workers were sparsely clothed and the pesticide from the leaking knapsack sprayers came into direct contact with the skin. Similarly, the workers knowing of the dangers of spraying into a head wind continued to do so even when the wind changed direction. ${ }^{7}$ The workers take these risks because reasonable alternatives are not readily available. Spray equipment is expensive, repair 
facilities are not easily accessible, or if they are, the cost is often prohibitive. Similarly, the farmer is often unable to wash himself or change his clothes after spraying owing to lack of facilities. Another factor contributing to episodes of poisoning among farmers in Sri Lanka is the tendency to use concentrations of pesticides in excess of the recommended dose $^{6}$ in order to be doubly certain of protecting their precious crops.

The use of pesticide mixtures by farmers is another problem area which needs to be considered. The highest case fatality for pesticide poisoning occurs among people poisoned with pesticide mixtures, ${ }^{5}$ possibly because of the difficulties in instituting appropriate treatment. Accidental pesticide poisoning not related to work is the other component of the $25 \%$ of cases of pesticide poisoning that require immediate preventive measures. It is often claimed that a large majority of such accidents are due to misuse of empty pesticide containers to store cooking oils, drinks, and other foodstuffs.

The control of these factors would contribute greatly to minimise unnecessary misery for a large number of people. It is an area where the agrochemical industry could take direct responsibility. For instance, it could take the necessary steps to provide suitable back up services for spray equipment, develop research on suitable protective clothing for use in tropical areas, promote farmers' awareness on the need to use proper concentrations of pesticide, stop marketing pesticide mixtures unless absolutely necessary, and, finally, develop pesticide containers that are less likely to be misused.

The ultimate responsibility for the control of pesticides must be devolved to national governments but unfortunately controls either do not exist or, where present, are poorly implemented. A recent study of control measures available in member countries of the FAO estimated that in 81 there were either no control measures or no information available, six countries were in the process of introducing some control measures, and 26 countries had control measures but implementation was poor (JAR Bates, paper at International Seminar on Controls of Chemicals in Importing Countries, Dubrovnik, 1981); most of these countries were from the Third World. Bull, commenting on the problem, states "Ideally Third World governments should enact strict legislative controls over the import, formulation, distribution, advertising, promotion, and use of pesticides. In addition, they should ensure adequate resources for effective enforcement of this legislation. Legislation should be complemented by an efficient and well trained agricultural research, training, and extension service especially geared to the needs of small and marginal farmers and farm labourers. Finally, a primary health care system should be closely allied to the agricultural extension services and include specifically occupational health care especially the prevention, recognition, and treatment of pesticide poisoning." strategies, and the activities in using a primary health care approach have been clearly set out by WHO in a document that states that the characteristics of such a health care system is based on the following, (1) accessibility, (2) relevance, (3) integration within the health system, (4) people's participation and their education, (5) cost effectiveness, and (6) collaboration with other sectors. ${ }^{8}$ It is the characteristics of this approach that make it the most appropriate and suitable form of health care for the nations of the Third World to embark on if they hope to contain the problem of acute pesticide poisoning.

Department of Social Medicine and Public Health National University of Singapore,

Outram Hill,

Singapore 0316.

\section{References}

' ILO Bureau of Statistics. Geneva: ILO, 1979.

${ }^{2}$ Steering Committee on Pest Control under the Conditions of Small Farmer Food Crop Production in Developing Countries. Report. Paris: OECD, 1977.

${ }^{3}$ Groupment International des Associations Nationales de Fabricants de Produits Agrochimiques. The manufacture and formulation of pesticides in developing countries. Brussels: GIFAP, 1983. (Technical monograph No 9.)

${ }^{4}$ World Health Organisation. Safe use of pesticides. Geneva: WHO, 1973. (Technical report series No 513.)

5 Jeyaratnam J, De Alwis Senewiratne RS, Copplestone JF. Survey of pesticide poisoning in Sri Lanka. Bull WHO 1982;60:615-9.

- Jeyaratnam J. Health hazards awareness of pesticide applicators about pesticides. In: Van Heemstra EAH, Tordoir WF, eds. Education and safe handling in pesticide application. Amsterdam: Elsevier, 1982:23-30.

'Bull D. A growing problem, pesticides and the world poor. Oxford: OXFAM, 1982.

${ }^{8}$ World Health Organisation. Primary health care and working populations. 1982, Geneva: WHO/OCH, 1982.

\section{Destruction of manuscripts}

From 1 July 1985 articles submitted for publication will not be returned. Authors whose papers are rejected will be advised of the decision and the manuscripts will be kept under security for three months to deal with any inquiries and then destroyed. 